\title{
DELIRIUM: INTERVENÇÕES APRESENTADAS AO PACIENTE EM UTI
}

\author{
DELIRIUM: INTERVENTIONS PRESENTED TO THE ICU PATIENT
}

\author{
Matheus Mendes Pascoal ${ }^{1}$ \\ Alexandre Casarin de Lima ${ }^{2}$ \\ Lucas Benedito Fogaça Rabito ${ }^{3}$ \\ Sabrina Ronconi Benedet Tashiro ${ }^{4}$
}

RESUMO: O delirium é uma das doenças mentais descritas na literatura médica, classificações psiquiátricas até o século XIX, foi redefinida como síndrome mais ampla. Afetando a consciência, desorientação. Este artigo tem objetivo de abordar pontos relevantes sobre o delirium com base na exposição dos estudos realizados e casos reais ocorridos em agravo da doença. Realizou-se um estudo bibliográfico das abordagens voltadas para a doença, juntamente com a opinião de pesquisadores, enfermeiros e médicos nas diversas formas correlacionadas e a equipe multiprofissional.

Descritores: Enfermagem. Delirium. Pacientes em UTI.

ABSTRACT: Delirium is one of the mental illnesses described in the medical literature, psychiatric classifications until the igth century. It was redefined as a broader syndrome. Affecting consciousness, disorientation. This article has aimed to address relevant issues of delirium based on the exposure of studies and real cases in aggravation of the disease. We conducted a bibliographic study of approaches focused on the disease, along with the opinion of researchers, nurses and doctors in different shapes and correlated the multidisciplinary team.

Descriptores: Nursing. Delirium. Delirium in ICU patients.

\footnotetext{
Acadêmico de Enfermagem do Centro Universitário Integrado (CEI). Lattes: http://lattes.cnpq.br/2047064166433787 Orcid: https://orcid.org/oooo-ooo3-4192-4579

2 Acadêmico de Psicologia do Centro Universitário Integrado (CEI). Lattes:http://lattes.cnpq.br/430400141991506I Orcid https://orcid.org/oooo-00o2-4307-218I

3 Graduado em Enfermagem pelo Centro Universitário Integrado (CEI). Residente em Urgência e $\begin{array}{lllll}\text { Emergência } & \text { Universidade de la } & \text { Londrina } & \text { (UEL). }\end{array}$ Lattes: http://lattes.cnpq.br/983630878832450o Orcid: https://orcid.org/oooo-ooor-8651-9193.

${ }^{4}$ Graduada em Enfermagem pela Universidade do Extremo Sul Catarinense (UNESC). Pós-Graduada no Curso de Especialização Multiprofissional em Saúde da Família pela Universidade Federal de Santa Catarina (UFSC), Pós-Graduada em Alta-Complexidade pelo Centro Universitário Integrado e Metodologias Ativas e Inovação no Ensino Superior (CEI). Mestre pelo Programa de Pós-Graduação em Ciências da Saúde pela Universidade do Extremo Sul Catarinense (UNESC). Doutoranda em Promoção da Saúde pela Universidade Centro de Ensino Superior de Maringá (CESUMAR).
} 


\section{INTRODUÇÃO}

É caracterizada como uma síndrome neurológica estudada na psiquiatria que definise como uma perturbação neurológica, associada pela falta de atenção, pensamento confuso, desorientação, muitas doenças e infecções podem originar-se podendo levar a demência e afetar a memória e cognição e motora. Pode desencadear ou ser confundida com outras doenças como a síndrome de Capgras que afeta a identificação do familiar, e o paciente experimenta essa fase não se recordando do ocorrido, ele considera as pessoas como desconhecidas. Pacientes com lesões cerebrais podem estar vinculados a danos neuroanatômicos (Feinberg, 2005; Devinsky, 2009; Darby e Prasad, 2016). O local mais comum afetado é lobo frontal direito, uma lesão pode ocasionar diversos danos ao paciente.

É comum em idosos, cujo cérebro tenha sido afetado por um acidente vascular cerebral, demência ou doença de Parkinson entre outras doenças degenerativas do sistema nervoso. Ambientes fechados como UTI podem contribuir para a formação do delirium, pois o isolamento leva a perda sensorial, pacientes podem ficar perturbados. $O$ sono perturbado pelos membros da equipe que despertam o paciente à noite, monitores altos, sinais sonoros, interface, vozes no corredor e alarmes podem agravar o estado clínico do paciente com delirium.

\section{METODOLOGIA}

Optou-se por uma revisão integrativa que consiste em um método de pesquisa utilizado com frequência na prática baseada em evidências, cujo objetivo é reunir e sintetizar os resultados anteriores.

Estabeleceu-se em seis passos: estabelecimento da questão de pesquisa; busca na literatura; coleta de dados; avaliação dos estudos incluídos; interpretação e discussão dos resultados e apresentação.

Estabeleceu-se o primeiro passo que norteou a pesquisa "Como o delirium pode impactar a vida do paciente sobre a perspectiva da visão de profissionais de saúde". Realizou-se a busca na literatura através de descritores: delirium and enfermagem. Os artigos encontrados foram entre os anos 2015 e 2021 utilizando-se como base de dados MEDLINE, LILACS, BDENF - Enfermagem, IBECS e Coleciona SUS. Os critérios de inclusão: texto completo, com os acessos nos idiomas portugueses, dissertações e teses. 
Selecionaram-se, após a leitura dos títulos e resumos, XXX artigos. Coletou-se os dados e informações, a identificação dos autores e coautores, objetivos do método, resultados principais do estudo.

\section{RESULTADOS E DISCUSSÕES}

Estudos recentemente realizados na literatura demonstram a alta incidência, o delirium que permanece subdiagnosticado cerca de $25 \%$ a $75 \%$, chegando a $100 \%$ em quadros clínicos caracterizados pelo estado hipoativo. Os fatores de riscos para maioria são modificáveis, pois medidas como remoção dos dispositivos invasivos, melhora na qualidade do sono, utilização de próteses podem ajudar o paciente.

A interrupção de sedativos até que o paciente desperte mostrou-se como uma estratégia que diminuiu o tempo de ventilação mecânica dos pacientes em UTI, o estudo inicial mostrou de 7,3 dias para 4,9 o tempo de internação em UTI. Na primeira interrupção o paciente mostrou a eficácia no aumento do número de dias livres de ventilação mecânica e redução no tempo de internação. O segundo mostrou uma melhora e reduziu o tempo de delirium em UTI. O protocolo de não sedação mostrou eficaz em manter os pacientes que estavam em ventilação sem sedativos algum e apenas com analgesia com morfina, caso apresentasse uma a agitação persistisse seria utilizado o propofol.

A relação de mortalidade e delirium em doentes críticos está relacionada, mas em seis meses as chances de morrer triplicam em indivíduos que apresentam essa cognição. $O$ tratamento de delirium é demasiadamente abrangente, o delirium é uma doença multifatorial necessita de intervenções multiprofissionais.

$\mathrm{O}$ atendimento multiprofissional e a mobilização, escala de Glasgow, Agitação, Dor, Orientação, Promoção do Sono, Ambiente e a família é essencial para o tratamento do delirium. Outro cuidado recomendado é a fisioterapia para pacientes críticos, pois melhora o quadro, diminui o tempo de ventilação mecânica e a internação hospitalar. A utilização de fármacos como haloperidol associados a um antipsicótico como olanzapina, risperidona ou quetiapina com doses de 0,5 a I mg a cada 08 horas. Houve redução do tempo do delirium pré 24 dias vs pós 27 dias. Na prática as intervenções são necessárias como o uso de antipsicótico. 
A participação da família na UTI pode trazer desafios a equipe, porém é benéfica para o tratamento do delirium e o desenvolvimento.

Pode ocorrer a associação do delirium e a confusão mental. A identificação é feita através da intervenção, saber o significado da doença é essencial. Pode apresentar três formas: hipoativo, hiperativo ou misto. No hipoativo os pacientes ficam confusos e desorientados e podem ser confundidos com depressão. No hiperativo ocorre a agitação e desorientação e alucinações é o mais tratado precocemente. No misto ocorre a flutuação da doença.

A participação da família é fundamental pois traz segurança ao paciente, no dia a dia da terapia existe o risco de o paciente lesionar-se. Por isso é fundamental que a contenção mecânica seja utilizada somente quando necessário. A redução do ruído, minimização da exposição à luz, temperatura agradável, a restrição de mobilidade e o isolamento social são medidas para a prevenção da condição.

Para o desenvolvimento do delirium fatores como idade superior a 70 anos, histórico de depressão, demência, perda visual ou auditiva, doença renal, hepática, HIV, consumo de álcool podem trazer riscos ao paciente para desenvolvimento dessa patologia.

O delirium é uma síndrome causada pelo comprometimento da atividade cerebral, devido a desorganização da atividade neural. Ocorre a alternância da consciência e atenção, déficits cognitivos, alterações de percepção, ilusões, alterações de humor. Frequentemente acompanhados com alterações, síndromes demenciais, condição médica, intoxicação com substâncias, abstinências de drogas entre outras definições. O diagnóstico do delirium continua sendo clínico, baseado na observação do paciente e no tratamento dos sintomas.

O tratamento médico é necessário, geralmente acomete pessoas idosas. Pode ocorrer com o recém-nascido rapidamente, i5 a 50\% das pessoas hospitalizadas sofrem com essa patologia. Em mais jovens o delirium é causado devido a utilização de medicamentos, o termo confusão é utilizado para pacientes que não conseguem processar informação adequadamente ou localizar, recordar de fatos. Caso a confusão desenvolva-se com lentidão pode originar a demência sendo necessária a assistência médica, caso não tratada leva a deterioração do estado mental.

A confusão é o sinal mais claro e reconhecido, pessoas jovens podem apresentar o delirium. A hospitalização é necessária quando apresenta outras doenças como 
desidratação, desnutrição e úlceras de decúbito. Lesões cerebrais focais podem levar a sintomas como ilusão de que um membro da família foi substituído por um impostor

O mapeamento de lesões desenvolvido pelos pesquisadores foi fundamental para verificar-se que as regiões de percepção, avaliação e familiaridade considerados como anormais foram identificados através de ressonâncias, e descobriu-se que as áreas do córtex retrosplenial esquerdo foi mais ativada nos estudos da ressonância magnética de familiaridade. O córtex frontal direito foi a região mais ativada na avaliação de crenças.

Portanto, uma única lesão pode gerar o agravo e levar a uma síndrome complexa que pode ser existente ou ocultada. Enfermeiros intensivistas podem melhorar o cuidado com o paciente com delirium com escalas específicas, avaliação da sedação e a avaliação direta do estado mental, atenção, pensamento e consciência.

O apoio emocional é essencial, pois sem ele não é possível realizar as medidas que são necessárias para prevenção e manejo. O desenvolvimento do delirium pode estar associado a utilização de medicamentos como propofol, meperidina, morfina, anticolinérgicos, antidepressivos, corticoides que mostraram que podem desenvolver delirium.

A sedação profunda é um problema em pacientes com ventilação profunda em UTI, a interrupção mostrou uma estratégia para redução da ventilação mecânica. Pacientes que apresentam delirium apresentam resultados negativos na evolução aumentando o tempo de hospitalização e ocorrência possível de mortalidade.

Em relação ao motivo de internação do delirium as complicações foram em decorrência da sepse, insuficiência ventilatória, epilepsia, escala de agitação e sedação de Richmond.

\section{CONCLUSÃO}

Os cuidados são essenciais, toda melhoria é fundamental para que o paciente com delirium venha obter os resultados esperados. O conhecimento sobre a doença $e$ as medidas preventivas podem facilitar a assistência de enfermagem, assim como a comunicação com os familiares e demais membros da equipe. É necessário realizar capacitações e atividades de educação continuada para os membros da equipe e o diálogo 
para a assistência à aplicação da escala diária para monitorar o delirium e auxiliando na diminuição dos custos hospitalares.

Esse estudo foi realizado em uma realidade específica de forma que os dados não podem ser generalizados mas podem contribuir para pesquisas. Pode-se perceber que para identificação do delirium devemos ter cuidados essenciais. O enfermeiro e a equipe multiprofissional possuem atividade privativas, autonomia profissional na atuação e gerenciamento. Garantindo a avaliação que detectam o delirium na beira do leito e aplicação nos pacientes.

O tratamento para o paciente com delirium pode ser realizado através da intervenção: estimular a mobilidade, autocuidado e independência, uso de calendários, relógios e esquemas de horários, evitar a mudança de ambiente e permitir o sono tranquilo,

A utilização de farmacológicos é indicada somente para pacientes agitados e com risco de segurança. Qualquer droga utilizada no tratamento terá efeitos psicoativos no estado de saúde mental, o agente mais utilizado é o haloperidol com dose inicial de o,5 a Img por via oral podendo repetir de 30 a 60 minutos, o objetivo é diminuir a agitação. Benzodiazepínicos são recomendados, mas podem causar sedação excessiva, além de alterar o estado de consciência.

\section{REFERÊNCIAS}

DARBY, R Ryan; LAGANIERE Simon; LEONE, Alvaro Pascual; PRASAD Sashank; FOX,Michael D. Finding the imposter: brain connectivity of lesions causing delusional misidentifications. Oxford Academic, Volume i40, Issue 2, Pages 497-507, 03/12/2016 Disponível em: DOI: https://doi.org/ro.1093/brain/aww288

SOUZA, Thieli Lemos; AZZOLIN, Karina de Oliveira; FERNANDES, Vivian Rodrigues. Cuidados multiprofissionais para pacientes em delirium em terapia intensiva: revisão integrativa. Revista Gaúcha de Enfermagem, Volume 39, Porto Alegre, 02/o8/2018, Scielo.DOI:http://www.scielo.br/scielo.php?script=sci_arttext\&nrm=iso\&lng=pt\&tlng=p t\&pid=Si983-I4472018000100505

Coutinho AFP, Lopes JL, Carneiro CS, et al. O Manejo Não Farmacológico do Delirium Sob a Ótica de Enfermeiros de uma Unidade de Terapia Intensiva Adulto. Rev Fund Care Online.2019. out./dez.; II(5):I242-I249. DOI: http://dx.doi.org/ro.9789/21755361.2019.viri5.1242-1249 
GOIS, Juliana; VIEIRA, Taiane; LOURENCO, Bruno da Silva; SOUZA, Daniel Ribeiro Soares de; VALENTIM, Luana; REIS, Lidiane. Assistência de enfermagem ao paciente com delirium na unidade de terapia intensiva: uma revisão integrativa / Nursing care for delirium patient in the intensive care unit: an integrative review / Atención de enfermería para pacientes de delirium en la unidad de atención intensiva: una revisión integrativa. Revista Nursing Online, Aprovado em 26/05/2019. Link: http://www.revistanursing.com.br/revistas/257/pg44.pdf

JUNIOR, Antonio Paulo Nassar. Impacto da sedação intermitente ou interrupção diária da sedação em pacientes sob ventilação mecânica / Daily sedative interruption versus intermittent sedation in mechanically ventilated critically ill patients. Dissertação USP, São Paulo, 2015.

Martins ,Juliana Bessa ${ }^{1}$, Santos, Adriana Alves dos ${ }^{1}$, Júnior Luís Joeci Jacques de Macedo $^{1}$, Eberle Carolina Chitolina ${ }^{1}$. AVALIAÇÃO DA PREVALÊNCIA DE DELIRIUM EMUMA UNIDADE DE TERAPIA INTENSIVA PÚBLICA. Revista Oficial do Conselho de Enfermagem. ARTIGO I3 v. Io, n. 3 (2019) Link: http://revista.cofen.gov.br/index.php/enfermagem/article/view/r759/578

FONTELA, Paula Caitano; JR, Luiz Alberto Forgiarini²; FRIEDMAN, Gilberto $^{1}$. Atitudes clínicas e barreiras percebidas para a mobilização precoce de pacientes graves em unidades de terapia intensiva adulto. Revista Brasileira terapia Intensiva, vol 30 no.2 São Paulo Apr/June 2018. Link:http://www.scielo.br/scielo. \&pid=So103-507X2018000200187

LEITE, Bruna Silva ${ }^{1}$; CAMACHO, Alessandra Conceição Leite Funchal ${ }^{2}$; QUEIROZ, Raquel dos Santos de 3 ; DIRÉ, Gláucio. A vulnerabilidade dos cuidadores de idosos com demência: revisão integrativa. Vulnerability of caregivers for the elderly with dementia: integrative review. Vulnerabilidad de los cuidadores de personas mayores con demencia: revisión integradora. Revista Online de Pesquisa Cuidado é Fundamental. Universidade do Rio de Janeiro. Jul/Sep 2017 Pag 888-892. Link: http://www.seer.unirio.br/index.php/ $/$ view/4513/pdf

SOUZA, Regina Cláudia da Silva Souza; BERSANETI, Mariana Davies Ribeiro; SIQUEIRA, Ellen Maria Pires; Meira, Luciana; BRUMATTI, Daiana Lepre, PRADO, Nilda Rosa de Oliveira. Capacitação de enfermeiros na utilização de um instrumento de avaliação de delirium. / Capacitação de enfermeiros na utilização de um instrumento de avaliação de delirium. / Nurses' training in the use of a delirium screening tool. Revista Gaúcha de Enfermagem. Hospital Sírio Libanês, Unidade de Terapia Intensiva, Serviço de Desenvolvimento de Enfermagem. São Paulo, São Paulo, Brasil vol.38 no.ı Porto Alegre 2017.Link:http://www.scielo.br/scielo.php?script=sci_arttext\&nrm=iso\&lng=pt \&tlng=pt\&pid=Sig83-I447201700010080I 
LUNA, Aline Affonsoㅁ, BRIDI, Adriana Carla ${ }^{2}$, SILVA, Roberto Carlos Lyra da. DELIRIUM EM TERAPIA INTENSIVA - UM ESTUDO RETROSPECTIVO DELIRIUM IN INTENSIVE CARE - A RETROSPECTIVE STUDY DELIRIO EN CUIDADOS INTENSIVOS UN ESTUDIO RETROSPECTIVO. Revista de Enfermagem UFPE On Line, Recife, 9(I):69-75, jan., 2015. Link: https://periodicos.ufpe.br/revistas/revistaenfermagem/article/vi/ıo308/ıo98o

PESSOA, Renata Fittipaldi; NÁCUL, Flávio Eduardo. DELIRIUM EM PACIENTES CRÍTICOS. Artigo de Revisão, Rev. bras. ter. intensiva I8 (2), Jun 2006. Link: https://www.scielo.br/j/rbti/a/3PLtXNyV9mKtfwdfZhFq6Yp/?lang=pt 